\title{
EHMTI-0099. Migraineur perception regarding family burden from chronic migraine: results of the CAMEO (chronic migraine epidemiology \& outcomes) study
}

\author{
DC Buse ${ }^{1 *}$, DW Dodick², AM Adams ${ }^{3}$ \\ From 4th European Headache and Migraine Trust International Congress: EHMTIC 2014 \\ Copenhagen, Denmark. 18-21 September 2014
}

\section{Introduction}

Chronic migraine $(\mathrm{CM})$ is associated with personal disability and burden; however the effect on family life is less certain.

\section{Aims}

The Family Burden Module (FBM) from the CaMEO study evaluated the extent, nature, and perceptions of burden of headache on migraineurs and their families.

\section{Methods}

CaMEO recruited persons to participate in a series of webbased surveys over 1 year to characterize migraine. The panel surveyed was sociodemographically representative of the US. FBM-Proband survey assessed 15 domains (134 items), including missed activities with family, partner, and children, and relationship impact. Data from respondents meeting CM study criteria (modified International Classification of Headache Disorders, 3rd edition, beta [ICHD-3b] migraine diagnosis $+\geq 15$ headache days/month for past 3 months; ICHD-3b criterion $\mathrm{C}$ not assessed) were included in current analysis.

\section{Results}

11,518 respondents had valid data from the FBM; 994 (8.6\%) probands were classified with CM. Probands reported reduced participation in family activities (6.9 days) and enjoyment of quality time with partners (6.6 days) within the preceding month. $20 \%$ reported missing vacations and reduced enjoyment at important family events
(9.4 weeks) within the previous year. Most probands felt their headaches made their partner's (64.1\%) and children's (56.5\%) lives hard, and thought they would be better partners $(72.5 \%)$ and parents $(59.1 \%)$ without headaches.

\section{Conclusions}

$\mathrm{CM}$ adversely affects family perception, relationships and activities. These findings suggest $\mathrm{CM}$ is a significant burden to migraineurs that extends to the family.

\section{Funding}

Allergan.

\section{Authors' details}

${ }^{1}$ The Saul R. Korey Department of Neurology, Albert Einstein College of Medicine and Montefiore Headache Center, Bronx, USA. ${ }^{2}$ Neurology, Mayo Clinic, Phoenix, USA. ${ }^{3}$ Global Medical Affairs, Allergan Inc., Irvine, USA.

Published: 18 September 2014

doi:10.1186/1129-2377-15-S1-D17

Cite this article as: Buse et al:: EHMTI-0099. Migraineur perception regarding family burden from chronic migraine: results of the CAMEO (chronic migraine epidemiology \& outcomes) study. The Journal of Headache and Pain 2014 15(Suppl 1):D17. 